\title{
The Impacts of Outreach Efforts by Research Computing at the University of Colorado Boulder
}

\author{
Thomas Hauser \\ Research Computing, University of \\ Colorado \\ 597 UCB \\ Boulder, CO 80309 USA \\ Thomas.Hauser@colorado.edu
}

\author{
Shelley Knuth \\ Research Computing, University of \\ Colorado \\ 597 UCB \\ Boulder, CO 80309 USA \\ Shelley.Knuth@ colorado.edu
}

\author{
Peter Ruprecht \\ Research Computing, University of \\ Colorado \\ 597 UCB \\ Boulder, CO 80309 USA \\ Peter.Ruprecht@colorado.edu
}

\begin{abstract}
An evaluation ${ }^{1}$ of services offered by Research Computing at the University of Colorado Boulder is presented. This evaluation is attempting to determine the impact of existing services, as well as of new services introduced based on user feedback and research on equivalent HPC groups at other institutions. The available user feedback suggests that most of these services have been wellreceived.
\end{abstract}

\section{CCS CONCEPTS}

- Outreach and Training $\rightarrow$ User support

\section{KEYWORDS}

\section{INTRODUCTION}

Research Computing (RC) at the University of Colorado Boulder provides services for researchers that include large-scale computing, storage of research data, high-speed data transfer

\footnotetext{
$1 *$ Produces the permission block, and copyright information

'The full version of the author's guide is available as acmart.pdf document It is a datatype.$$
\text { (c) (i) }
$$$$
\text { This work is licensed under a Creative Commons Attribution International 4.0 License. }
$$

PEARC17, July 09-13, 2017, New Orleans, LA USA

(C) 2017 Copyright held by the owner/author(s). ACM ISBN 978-1-4503-52722/17/07

http://dx.doi.org/10.1145/3093338.3093377
}

capabilities, and support for data sharing. These resources include the on-campus supercomputer, named Rocky Mountain Advanced Computing Consortium (RMACC) Summit, which came online in January 2017 and includes approximately 500 nodes [1]; a largescale data storage resource called the PetaLibrary; a visualization cluster; and a condo cluster. The group also provides consulting in computational science and data management [2]. In addition, RC offers a variety of outreach and training efforts that are intended to assist users with understanding high performance computing (HPC).

While the main focus of Research Computing is to provide HPC infrastructure to the broader University of Colorado Boulder community, the utilization of this infrastructure is in no small part impacted by how well users understand how to utilize it. Many of RC's services revolve around ensuring that users have all the tools available at their disposal to understand the nuances of RC's HPC infrastructure and supercomputing in general. Other services involve a community outreach component, which serves to both introduce Research Computing to campus entities as well as to communicate changes to our infrastructure.

The purpose of this project is to explore the offerings from Research Computing to better understand their impact on RC users. The project period extends from January 2016 through June 2017. This project examines several existing services, as well as new services that were developed in the hope of better meeting users' needs. The creation of new services was motivated in part by tendencies in the tickets $\mathrm{RC}$ received and feedback from participants in two user focus groups that $\mathrm{RC}$ facilitated. Additional ideas for improvement came from studying similar HPC groups at institutions that were fellow members of RMACC to determine if we could adopt their services, and the effect that these new services would have on our users. Users provided a limited amount of feedback on RC services, which aided RC in evaluating our efforts. This paper will briefly discuss our services, 
The Impacts of Outreach Efforts by Research Computing at the University of Colorado Boulder

the steps we took to evaluate these services, and the preliminary results of these efforts.

\section{AVAILABLE SERVICES}

The approach to this study was three-fold. First, we chose several of RC's existing offerings to study and explore evaluative methods by which we could determine if there was appropriate buy-in from users. Second, we explored new services that could be added to assist users. These new services were identified in three ways: by holding user focus groups, by researching equivalent HPC groups at other institutions to determine additional services that had been successful in reaching users, and by considering topics on which users had submitted tickets. Third, we evaluated the services to identify how users were being impacted.

\subsection{Existing Services}

The existing services in place at the beginning of the study period (January 2016) included:

1. Meetups: short training sessions that provided a brief overview of relevant HPC topics. Topics included "Introduction to Linux", "How to Use a Supercomputer", and "Python in HPC". These sessions were one hour long and offered weekly over lunch. The Meetups had been in place for approximately three years prior to this study. Attendance had peaked in 2013-2014, but had waned significantly since. While specific numbers are not available, anecdotal evidence from Knuth (who has managed the Meetups since 2014) noticed that average attendance was approximately 20 people (the maximum allowed in the room) in 2014, but had dropped to approximately three to five per session by $2015-2016$.

2. Blanca condo cluster: researchers can purchase their own nodes on this cluster through RC. RC configures, networks and maintains the cluster.

3. User support: $\mathrm{RC}$ staff provides direct assistance to users in two possible ways: by answering questions through the online ticketing system, and by providing one-on-one assistance.

4. Showcases: RC gives tours of the supercomputer that are free and open to the public. Interviews with prominent researchers who use $\mathrm{RC}$ resources are posted on the RC website. The group further increases its visibility by utilizing social media accounts, as well as campus and department newsletters.

5. Presentations at faculty meetings: In 2015, RC staff visited $48 \%$ of the departments and institutions on campus at their regular department meetings, and repeated most of these visits in late 2016. The purpose
PEARC'17, July 2017, New Orleans, Louisiana USA

of this outreach was to introduce RC and the services the group provides to potential new users. RC staff also visited several postdoc and graduate student groups on campus.

6. Online documentation: the RC website provides users with text-based documentation on using RC resources, including a Getting Started guide for new users and a more comprehensive User Guide. The website directs users for whom the online documentation is insufficient to submit tickets.

Of the six services listed, we chose two to explore further as a component of this study: the meetups, and the condo cluster. We chose these two services in particular because they are relatively high profile services that are not necessarily a default service that would be offered as part of a research computing group. The other services offered are either integral parts of our services that are well-established, or lacked sufficient information to complete a comprehensive study. One exception to both of these cases is RC's online documentation. The results of an evaluation of the online documentation are not included in this paper because it is ongoing. We will discuss some of this work in Section 4.

\subsection{Additional New Services}

Potential new services were identified in several ways. The first was based on ticket topics. Despite the availability of text-based documentation for new users on the RC website, many new users submitted tickets because they did not understand the steps they needed to take to begin using RC resources. Tickets from 2015, as well as tickets submitted throughout the study period, were of interest because the new service expected to have an impact on ticket topics, the New User Seminar, was not introduced until the end of the study period. Out of 2,777 tickets submitted between February 2, 2015 and June 1, 2017, 31\% contained issues typically relevant to new users. These issues included obtaining an allocation, establishing two-factor authentication, and job submission (Figure 1). After noticing these trends, RC made efforts to gather additional information to determine specific ways we could improve services.

The second was research on services offered by equivalent HPC groups at other RMACC institutions. Several RMACC institutions offered short trainings similar to RC Meetups, which taught specific skills in isolation. Another popular form of training was multi-day workshops geared towards either beginning or advanced users. One of these workshops was a three-day "Bootcamp" for beginning users at the University of Wyoming [3]. In addition to in-person training, most institutions provided online resources for new users, including short how-to videos. Fellow RMACC institutions also emphasized their efforts to showcase their resources on campus and reach out to faculty and department heads in order to attract new users [4]. 
The Impacts of Outreach Efforts by Research Computing at the University of Colorado Boulder

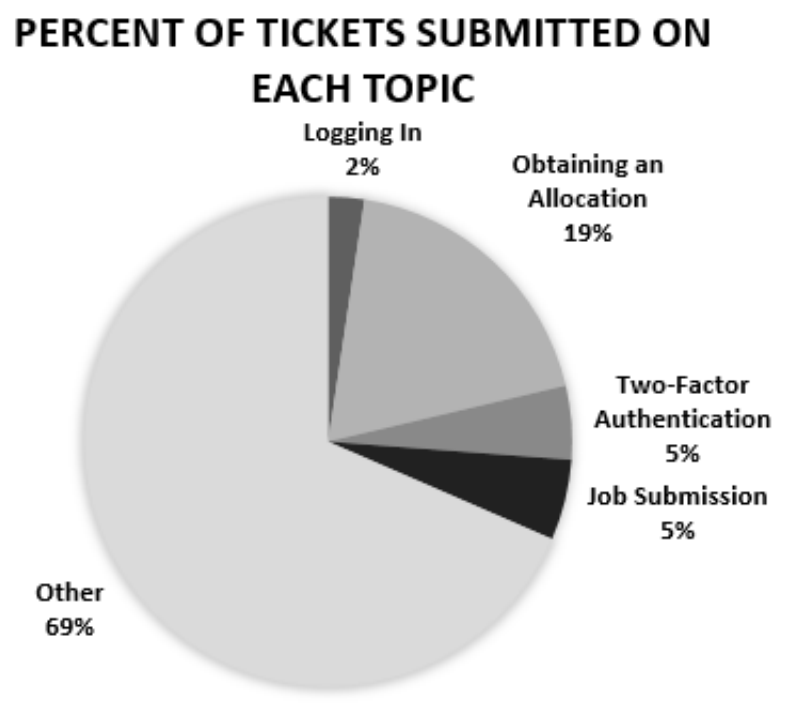

Figure 1: Topics of tickets submitted to RC suggesting that beginner users needed guidance beyond the documentation available online.

The third was from two focus groups held in December 2016. The focus groups were valuable in that they provided information not only about potential new services RC could offer, but also about the impact of existing services. One focus group was made up of five advanced users, and the other included three beginning users. Participants in the focus groups suggested making individual, in-person help available, offering training workshops for new users multiple times each semester, and improving website documentation. The idea of holding a focus group came partway through the study, which is why we did not hold the event until December 2016.

As a result of this information, the services added or modified were the following:

1. A two-day "Basics of Supercomputing Bootcamp" inspired by the annual workshop at the University of Wyoming, which was designed to teach beginner users the skills needed to work on large-scale compute resources. Many researchers need large-scale supercomputing systems to complete their studies, but have little knowledge to do so effectively or properly. This workshop was created with these users in mind, to not only ensure they understand how to use the system to produce accurate results, but understand the nuances of utilizing shared resources. The first Bootcamp was offered in July 2016, and the second was offered in January 2017. The Bootcamps were evaluated based on attendance numbers and the results of post-
PEARC'17, July 2017, New Orleans, Louisiana USA

surveys taken by attendees.

2. A group of students called the Student Cohort was created to address more advanced users' needs for inperson assistance to resolve the problems they encountered when using Research Computing resources. The Cohort would meet with RC users who had more complex issues to provide more interactive one-on-one support. The cohort consisted of two students who initially had introductory HPC skills, and were trained by RC staff both in advancing those technical skills as well as in soft skills (e.g., how to speak to new users effectively). The Cohort was evaluated by reaching out to individuals the Cohort had helped and asking them about their experiences.

3. Following on the success of the Blanca condo cluster, the Summit condo cluster option was provided to RC users. For a limited time, CU researchers were given the opportunity to purchase additional nodes to be integrated into Summit. These nodes, maintained by RC staff, allowed users to receive increased queue priority proportional to their purchase.

4. A new workshop, called the New User Seminar, was created for new users to learn the most basic skills needed to use RC resources. The New User Seminar was created based on the large percentage of tickets received regarding elementary questions by new users (detailed in the previous section). This seminar covers topics such as the steps required to use RC resources (getting an account, setting up two factor authentication, and applying for allocations), how to log in, node types, how to transfer data, how to install software, and template scripts for job submission. Participants could attend these monthly two-hour workshops either in person or online. The first three New User Seminars were held in March, May and June 2017.

5. Multiple changes were made to the Meetups in the hopes of increasing attendance numbers. A web streaming capability was added to Meetups in 2016. More advanced topics than had previously been available, such as "Advanced OpenSSH Client Configuration" and "Fortran for Experienced Programmers", were introduced to the Meetup lineup in the fall 2016 semester. In the spring 2017 semester, additional changes were introduced: Meetups were offered at 4:00pm rather than over lunch, email reminders were sent to members of the online Meetup group an hour before the training began, and Meetups were offered biweekly rather than weekly. Advanced topics were offered as well as multiple talks that were specific to using the new supercomputer, Summit. 
The Impacts of Outreach Efforts by Research Computing at the University of Colorado Boulder

\section{DISCUSSION}

The conclusions drawn about RC services during this project were limited in part by users' willingness to provide feedback. Surveys and emails throughout the project had persistently low response rates, meaning that the needs and impressions that were common among survey respondents were not necessarily common throughout the user base. Another obstacle to drawing firm conclusions about the effectiveness of RC services was the fact that multiple changes were introduced to services simultaneously, as in the cases of the Meetups and the second Basics of Supercomputing Bootcamp, meaning that no one change could be isolated as having a causal relationship with training attendance or with users' levels of satisfaction. However, user feedback on the simultaneous changes was still helpful in forming an impression of whether the combined changes had been well-received. Despite the lack of firm conclusions, receiving feedback from users enabled RC to gain valuable insight into their perceptions of our services, which we had previously lacked. Even anecdotal feedback was helpful in the process of determining whether RC's impression of our services aligned with those of users.

1. The "Basics of Supercomputing Bootcamp" workshops were evaluated based on attendance numbers and survey responses. The first bootcamp, held in July 2016, was offered for free. The workshop was limited to 50 participants, and 39 attended, filling $78 \%$ of the available seats. $28.2 \%$ of attendees completed the Bootcamp post-survey. $90.9 \%$ of respondents reported that attending the bootcamp caused them to either make an account with RC or increase their usage on an existing account. These respondents also submitted comments about adjustments that should be made to the workshop agenda, which were used to create a slightly modified agenda for the January 2017 workshop. Because the staff teaching the July workshop had felt that they were straining themselves to help all 50 participants, the January workshop was limited to 30 participants to ensure that all participants would receive the help they needed from the teaching staff. Participants were charged $\$ 30$, which went towards the cost of food for the workshop and was intended to incentivize registrants to attend the workshop. Thirty people paid and registered, and 22 of those attended, filling $73 \%$ of the available spots. $36.4 \%$ of attendees responded to the Bootcamp post-survey. The postsurveys for both workshops asked attendees to rate the workshop's effectiveness in teaching each of several skills. For the five major skills that were taught at both workshops, an average of $90.91 \%$ of participants in the July 2016 workshop reported that the workshop had been "very effective" or "mostly effective" in teaching the skill; for the January 2017 workshop, that figure was $92.5 \%$ (Figure 2). $100 \%$ of respondents indicated that attending the Bootcamp caused them to either make an
PEARC'17, July 2017, New Orleans, Louisiana USA

account or increase their account usage. The fact that nearly all spots were filled at the first workshop and that all spots were filled during registration at the second, smaller workshop suggested that the Bootcamps met a training need for a portion of users. Knuth has also received multiple requests anecdotally to offer the workshop more often. While higher response rates would have made the survey results more conclusive, survey responses from both Bootcamps indicated that attending Bootcamps met new users' needs by teaching them new skills and increasing their usage of Research Computing resources.

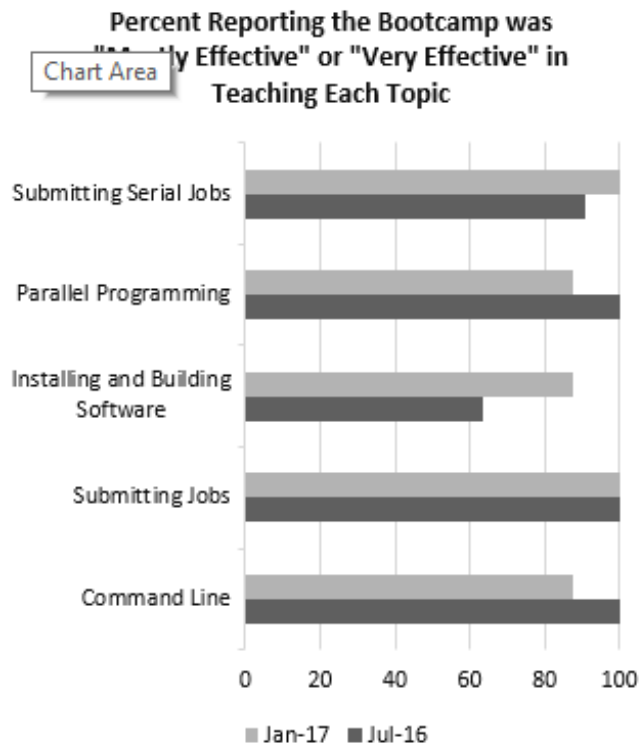

Figure 2: Results from surveys given to the "Basics of Supercomputing Bootcamp" participants to determine the effectiveness of individual topics at the two workshops.

2. To evaluate the Student Cohort, we reached out via email to the ten individuals who had received help from the Cohort. Three of these individuals responded to emails. One of the respondents had been in contact with a member of the Student Cohort, but the student was still in the process of helping him resolve his issue. That respondent felt that in-person help was a valuable service, especially for new users, and reported that the student had already been helpful in giving him an overview of Summit nodes. The second respondent enthusiastically praised the student who had helped him, saying that the student had been flexible and enthusiastic in addition to his technical expertise, and had resolved the user's issues. The third respondent reported that their issue had been resolved and that they had been pleased with the speed and effectiveness of the 
The Impacts of Outreach Efforts by Research Computing at the University of Colorado Boulder

help received. These three responses suggested that the Student Cohort was fulfilling its purpose of helping users resolve specific computing issues, although the two responses alone did not provide enough information about users' experiences with the Student Cohort for a firm conclusion to be drawn.

Another metric of success of the Student Cohort has been in its ease of RC staff duties. The RC group is small, and does not have the time to meet with all users in person to answer their questions. The introduction of the cohort has freed up time for RC staff to work on other support tasks. In addition, training two promising students in HPC technical and soft skills has had a broader impact on these students individually.

3. New User Seminars were evaluated based on attendance numbers and survey results. Five people attended the first New User Seminar in person in March 2017 and six attended online. The second seminar, in May 2017, was attended by three in-person participants and two online participants. Nine people attended the June 2017 seminar in person and five attended online. 97\% of survey respondents from all three seminars rated the training as "good" or "very good" in categories related to the appropriateness of the content and the overall instructor. $93 \%$ of respondents agreed or strongly agreed with the statement that they would recommend the training to others. Results are too early yet to determine if a drop in tickets has been seen since the introduction of this seminar.

4. The condo clusters (Blanca and Summit) were evaluated based on the number of nodes purchased, as well as some feedback from condo cluster users. In 2015, five research groups purchased 47 nodes. We completed a condo purchase in late 2016, and we are beginning to study these results. Further evaluation of the condo clusters came from surveying users. Out of fifteen condo partners from both Blanca and Summit, five responded to the survey. They cited their ability to avoid waiting in the queue to run jobs on Summit and the nodes' high-memory capabilities as reasons the availability of the condo cluster improved their experiences. Two of the five respondents indicated that they would not have used Research Computing resources if the condo cluster had not been available. While the evidence of the condo cluster's benefits in this survey came from a small group, it did indicate that the condo cluster met the needs of users who wanted to use high-memory nodes and avoid longer waits in the Summit queue.

5. Meetups were evaluated based on trends that Knuth observed in attendance and results from surveys issued to attendees at the end of a workshop. Because
PEARC'17, July 2017, New Orleans, Louisiana USA

attendance at Meetups had steadily been decreasing since 2015, group members were surveyed to determine whether they attended Meetups and, if not, what would cause them to attend more Meetups. The survey respondents who did not attend Meetups cited several reasons: Meetups did not teach the skills they wanted, they did not regularly check the Meetup website, they were not available when Meetups were offered, or whether they attended depended on the content of the Meetup. With an only 3\% response rate from the 648member online Meetup group, it was impossible to generalize the survey results to draw conclusions about what most attendees would want. Due to the low survey response rate, RC made a further effort to gather information by reaching out to infrequent attendees, defined as members of the Meetup group who had been active on the Meetup website within the past three months but had attended fewer than five Meetups. Seventeen of these infrequent attendees responded to emails. Eight respondents said that they were interested in Meetup topics but had been too busy to attend; seven said they wanted to attend Meetups but were unable to attend due to either the time or location of Meetups (Figure 3).

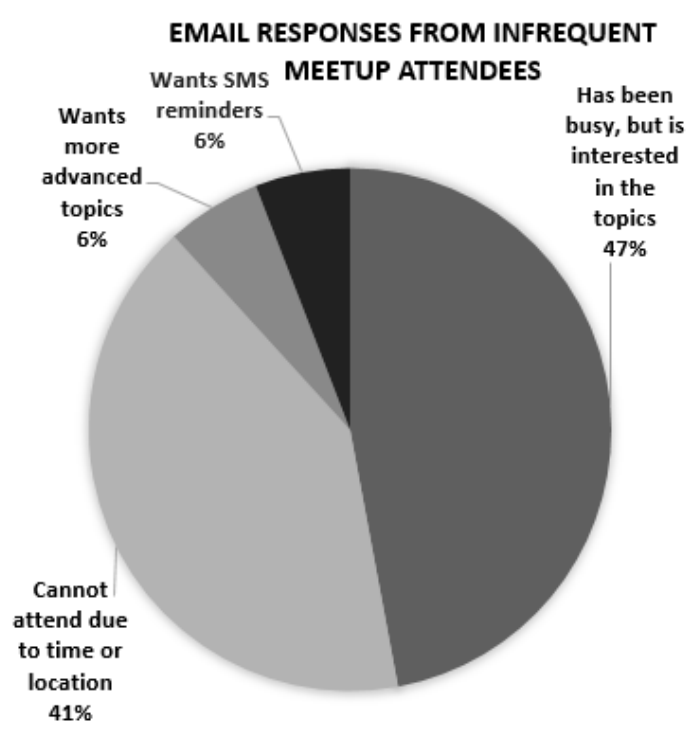

Figure 3: Email responses from infrequent Meetup attendees suggesting that changing the time, location and topics of Meetups would increase attendance

While feedback from Meetup group members was limited, RC incorporated the feedback it received into ideas for potentially improving Meetup attendance, which included:

a. Hold Meetups at a time other than lunch

b. Hold Meetups on different topics. Suggestions included 
The Impacts of Outreach Efforts by Research Computing at the University of Colorado Boulder

more advanced versions of Meetups that are already offered at an introductory level

c. Provide food at Meetups

d. Increase awareness about the fact that users can attend Meetups online via streaming (a new service offered in 2016)

e. Notify Meetup group members about upcoming Meetups

Based on these ideas, Research Computing made several changes during Fall of 2016 and Spring of 2017. In Fall 2016, RC offered more advanced topics rather than introductory topics for beginners, as had been previously offered. This adjustment did not cause any notable changes in attendance. RC also added web streaming to the Meetups, and saw a moderate increase (anecdotally, three to four additional people on average per Meetup). In Spring 2017, RC increased the adjustments to include: biweekly rather than weekly meetings, changing the time to $4 \mathrm{pm}$ rather than over lunch, and reminding members of the upcoming meetup one hour prior via email. The topics during early Spring 2017 were centered around the use of the new Summit supercomputer, while topics later in the semester were similar to the caliber of those offered during in Fall 2016. Although the number of RSVPs on the Meetup website remained low, attendance numbers surged at the Summit-related Meetups, reaching levels seen in 2014. Because multiple changes were introduced simultaneously, we were unsure which led to the increase in attendance. However, later in Spring 2017, after the talks related to the new Summit supercomputer were over, attendance numbers declined again, returning to the low levels observed in 2015-2016. In the absence of recorded attendance data, we relied on Knuth's observations as the best available method of determining whether making changes to the Meetups had been effective in increasing attendance numbers. While Summit-related Meetups drew larger audiences, the changes did not produce a consistent increase in the number of attendees at traditional Meetups that taught individual HPC skills. Because so few users were benefiting from Meetups, we decided to discontinue Meetups for the 2017-2018 academic year. RC will revisit this for the following academic year. Based on increases in attendance, it might make sense to offer more Summit-focused talks.

$\mathrm{RC}$ also offered other services that weren't specifically studied as part of this work, but resulted from some of the feedback received either in surveys from other workshops, tickets, or the focus groups. One of these was to add additional, more advanced workshops to the training suite available to RC users. These workshops are usually longer than the Meetups (sometimes several days or hours), and provide a more in depth look at a topic. Some examples of these workshops have been "Parallel Computing with Matlab, Python, and R", "Interactive Data Visualization and Analysis with Python and Bokeh", or "Programming with Python". Industry partners, such as Intel and NVIDIA, have also offered workshops through the RC group at CU Boulder. Additionally, we have brought workshops created at
PEARC'17, July 2017, New Orleans, Louisiana USA

other institutions to our university, such as the Software Carpentry series or the XSEDE HPC Monthly Series. The results of these workshops, and a comprehensive study of their impact on our users has not been completed, but interest has been high thus far in these workshops, which has totaled nearly ten since January 2016.

\section{LESSONS LEARNED AND FUTURE WORK}

This study seeks to determine the impact of various services offered by the Research Computing group at the University of Colorado Boulder. In particular, it aims to determine the impact of several existing services as well as new services added based on user input and research on equivalent HPC centers. The feedback currently available from users suggests that new services including the Basics of Supercomputing Bootcamps, New User Seminars, the Student Cohort and the condo component of the Summit cluster were well-received. These services have now become regular offerings of the $\mathrm{RC}$ group. Because changes to Meetups did not appear to meet the goal of increasing attendance numbers, Meetups will be discontinued in the 2017-2018 academic year. Further results are being studied.

In the process of working on this project, we learned several lessons:

1. Drawing conclusions about RC services will require more rigorous data collection efforts than were undertaken during this project. For example, collecting Meetup attendance numbers would have improved our ability to determine whether the changes we introduced were impacting attendance numbers. Leaving the last five minutes of all in-person trainings as a designated time when all participants filled out in-person surveys and collecting the surveys as exit tickets would have increased the number of survey responses available to evaluate trainings. Holding more focus groups would have given us more confidence that the consensus reached in the focus groups were prevalent in the user base. Asking that users submit surveys immediately after receiving a service, such as help from the Student Cohort, would likely yield higher response rates than asking all users who had been helped to give us feedback at the moment it became relevant to our research.

2. Researching similar HPC groups at other institutions was helpful in designing new services when we believed that our users' needs were not being met but were unsure of which specific services to introduce to meet them. The inspiration for the Basics of Supercomputing Bootcamp, a successful service for new users, came from another HPC group.

3. Our ability to provide training is limited by users' 
The Impacts of Outreach Efforts by Research Computing at the University of Colorado Boulder

desires to improve their skills. In the case of the Meetups, the available feedback suggested that many Meetup users believed the skills taught in Meetups would be valuable to them, but they did not attend Meetups even when the time was changed to encourage attendance. Average attendance of fewer than five members of a 648-person online group suggested that attending Meetups was not a priority for most members of the group even if attending the Meetups would have made them more skilled RC users. Participants in the focus group of advanced users explained that many users see a trade-off between person time and computing time: they would rather use RC resources inefficiently to save their personal time for research work than spend their time optimizing their applications or workflows for efficiency. The fact that users are lacking in a certain skill area is not sufficient evidence that RC should devote resources to teaching those skills; more evidence from users about which trainings are sufficiently high-priority for them to attend is necessary.

4. One way to combat training fatigue, which was suggested in one of the focus groups, is to offer videos of either training or of topics on the web. RC posts videos of trainings on the RMACC YouTube channel. Advertising these and tracking the number of views and downloads would help determine the impact on users.

$\mathrm{RC}$ is actively continuing to improve our services beyond what was studied in this paper. Ongoing and future work includes the following:

1. Issues with the $\mathrm{RC}$ website were a recurring theme in the focus groups, as well as anecdotally in conversations with RC staff. In Spring 2017, a CU Business School course did an evaluation of the RC website and online documentation, showing that a number of improvements were needed. RC staff are currently mapping a new plan for the website, which includes updated and increased documentation. RC users in the focus groups also indicated a desire for short (less than five minute) videos on the website on specific topics. The authors are in the midst of recording these videos to be put on the web in Summer 2017.

2. Improving surveying techniques, both to obtain more responses and to collect demographic information on $\mathrm{RC}$ users and workshop participants, which is currently unavailable. Determining whether certain demographics are underrepresented in $\mathrm{RC}$ workshops or in the user base will help RC determine how we can work to increase diversity.
PEARC'17, July 2017, New Orleans, Louisiana USA

\section{ACKNOWLEDGMENTS}

This work was partially supported by the University of Colorado Boulder and NSF Grant ACI-1532236.

\section{REFERENCES}

[1] Anderson, J., P.J. Burns, D. Milroy, P. Ruprecht, T. Hauser, and H. J. Siegel, 2017: Deploying RMACC Summit: An HPC Resource for the Rocky Mountain Region. In Proceedings of PEARC17, New Orleans, LA, USA, July 09-13, 2017, 7 pages. DOI: 10.1145/3093338.3093379

[2] Knuth, S.L., A.M. Johnson, and T. Hauser, 2015: Research Data Services at the University of Colorado Boulder. Bull. Assoc. Information Sci. and Tech., 41(6), 35-38.

[3] Luecke, Glenn, Personal Communication, April 8, 2016

[4] Cleveland, S., B. Freeman, C. Koch, L. Michael, A. Orendt, and E. Shaw, "ACI-REF Best Practices of Facilitation." ACI-Ref Best Practices of Facilitation. National Science Foundation, n.d. Web. 31 Aug. 2016. 